\title{
ORIGINAL RESEARCH \\ Blood-Flow Characteristics in a Terminal Basilar Tip Aneurysm Prior to Its Fatal Rupture
}

D.M. Sforza

C.M. Putman

E. Scrivano

P. Lylyk

J.R. Cebral
BACKGROUND AND PURPOSE: The development and validation of methods to stratify the risk of rupture of cerebral aneurysms is highly desired because current treatment risks can exceed the natural risk of rupture. Because unruptured aneurysms are typically treated before they rupture, it is very difficult to connect the proposed risk indices to the rupture of an individual aneurysm. The purpose of this case study was to analyze the hemodynamic environment of a saccular aneurysm of the terminal morphology subtype that was imaged just before its rupture and to test whether the hemodynamic characteristics would designate this particular aneurysm as at high risk.

MATERIALS AND METHODS: A patient-specific CFD model was constructed from 3DRA images acquired just hours before the aneurysm ruptured. A pulsatile flow calculation was performed, and hemodynamic characteristics previously connected to rupture were analyzed.

RESULTS: It was found that the aneurysm had a concentrated inflow stream, small impingement region, complex intra-aneurysmal flow structure, asymmetric flow split from the parent vessel to the aneurysm and daughter branches, and high levels of aneurysmal WSS near the impaction zone.

CONCLUSIONS: The hemodynamic characteristics observed in this aneurysm right before its rupture are consistent with previous studies correlating aneurysm rupture and hemodynamic patterns in saccular and terminal aneurysms. This study supports the notion that hemodynamic information may be used to help stratify the rupture risk of cerebral aneurysms.

ABBREVIATIONS: CFD = computational fluid dynamics; 3DRA $=3 \mathrm{D}$ rotational angiography; $\mathrm{MCA}=$ middle cerebral artery; $\mathrm{SAH}=$ subarachnoid hemorrhage; WSS $=$ wall shear stress
$\mathbf{T}$ he development and validation of methods to stratify and assess the rupture risk of cerebral aneurysms are highly desired because current treatment risks can exceed the natural risk of rupture. ${ }^{1-3}$ Earlier studies based on patient-specific CFD have compared hemodynamic characteristics between ruptured and unruptured saccular aneurysms and found that high-rate asymmetric inflows, concentrated inflow jets with small impaction zones, elevated maximum WSS, and complex unstable flow patterns were correlated with clinical history of previous rupture. ${ }^{4-6}$ Because unruptured aneurysms are typically treated before they rupture, it is very difficult to connect the proposed risk indices to the rupture of an individual aneurysm. This article presents a case study of a saccular aneurysm of the terminal morphologic type that was imaged with $3 \mathrm{D}$ angiography a few hours before its rupture. This study provides the rare opportunity to analyze the hemodynamic environment at the time of rupture and to test whether previously identified risk indices or characteristics would designate such aneurysm as high risk.

Received July 16, 2009; accepted after revision October 23.

From the Department of Computational and Data Sciences (D.M.S., J.R.C.), Center for Computational Fluid Dynamics, George Mason University, Fairfax, Virginia; Department of Interventional Neuroradiology (C.M.P.), Inova Fairfax Hospital, Falls Church, Virginia; Department of Neurosurgery (C.M.P.), George Washington University School of Medicine, Washington, DC; and Departments of Neurosurgery and Interventional Neuroradiology (E.S., P.L.), Clinica Sagrada Familia, Buenos Aires, Argentina.

We thank Philips Medical Systems for financial support. J.R.C. also acknowledges support from the National Institutes of Health grant R01NS059063.

Please address correspondence to Juan R. Cebral, PhD, Center for Computational Fluid Dynamics, 4400 University Dr, MSN 6A2, Fairfax, VA 22030; e-mail: jcebral@gmu.edu

Indicates open access to non-subscribers at www.ajnr.org

DOI 10.3174/ajnr.A2021

\section{Materials and Methods}

\section{Clinical and Imaging Data}

A 59-year-old female patient presented with headaches and $\mathrm{SAH}$ (Fisher III and Hunt-Hess II). CT showed 3 cerebral aneurysms, 2 in the right MCA and 1 at the tip of the basilar artery. These images also showed that a 7.5-mm aneurysm on the right MCA had ruptured. This aneurysm was then treated via endovascular embolization with coils. The basilar artery aneurysm was left untreated, and the patient presented a few hours later with a new SAH (Fisher IV). A CT scan showed new blood in the vascular territory corresponding to the basilar artery with intraventricular bleeding (Fig 1). No blood in the region of the coiled MCA aneurysm was observed in these images. Thus, it was determined that the second hemorrhage was due to the rupture of the basilar tip aneurysm. Unfortunately, the patient then died.

During the endovascular procedure, catheter angiograms were obtained by standard transfemoral catheterization of the cerebral vessels, and digital subtraction imaging was done on an Integris Biplane Unit (Philips Medical Systems, Best, the Netherlands). These images were obtained during a 10 -second injection of contrast agent and a $180^{\circ}$ rotation with imaging at 15 frames per second for a total of 8 seconds. The corresponding 120 projection images were reconstructed on a dedicated Phillips workstation into a 3D dataset of $256 \times 256 \times 256$ isotropic voxels covering a FOV of $25 \mathrm{~mm}$ in all 3 directions. The dataset was exported into a personal computer for vascular and hemodynamic modeling. A 3DRA image of the aneurysm is shown in Fig 2 (left panel).

\section{Hemodynamics Modeling}

A patient-specific CFD model of the aneurysm was constructed from the 3DRA image by using previously developed methods. ${ }^{7}$ The image was filtered, and segmentation was performed with a seeded region- 


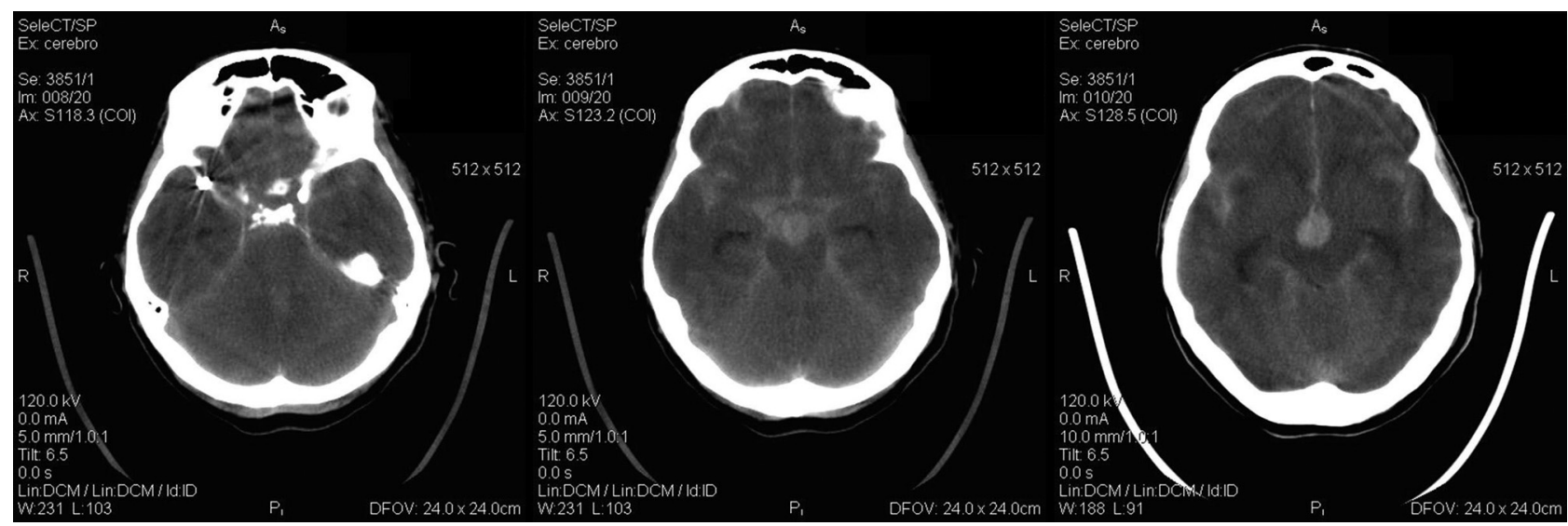

Fig 1. CT scans demonstrating the bleeding with intraventricular hemorrhage of the basilar tip aneurysm and no extra blood in the region of the coiled MCA aneurysm.

growing algorithm to reconstruct the topology of the vascular network followed by an isosurface deformable model to adjust the geometry to the vessel boundaries. ${ }^{8}$ The vascular model was then smoothed with a nonshrinking algorithm, ${ }^{9}$ and vessels were truncated perpendicularly to their axes. The final geometric model obtained is shown in Fig 2 (right panel). A volumetric grid composed of tetrahedral elements was generated by using an advancing front method with a resolution of $0.15 \mathrm{~mm}$, resulting in approximately a 3.2 million element mesh. Blood flow was considered an incompressible Newtonian fluid (with attenuation $\rho=1.0 \mathrm{~g} / \mathrm{cm}^{3}$ and viscosity $\mu=0.04$ poise) and modeled by the unsteady Navier-Stokes equations in $3 \mathrm{D} .{ }^{10}$ These equations were numerically solved by using an implicit finite-element formulation on unstructured grids. ${ }^{11,12}$ Pulsatile physiologic flow conditions derived from phase-contrast MR imaging flow measurements on healthy subjects were prescribed at the inlet boundary (basilar artery) by using the fully developed Womersley profile and scaled with the inlet area to achieve a mean WSS of $15 \mathrm{dyne} / \mathrm{cm}^{2}$ at the inlet. $^{13,14}$ The flow division from the basilar artery to the outflow vessels was determined from their area ratio, and corresponding pressure boundary conditions were applied at the model outlets. Vessel walls were assumed rigid, and no-slip boundary conditions were applied at the walls. A total of 2 cardiac cycles were computed by using 100 time steps per cycle; the results for the second cycle are presented. Because we were interested in gross characteristics of the blood flow pattern, this number of time steps was enough.

\section{Data Analysis}

The numeric results were visualized and inspected to classify the aneurysm according to hemodynamic characteristics previously identified as potential rupture risk factors. ${ }^{4-6,15}$ Namely, we considered the following characteristics: 1) location of the flow impaction (neck, body, or dome); 2) size of the flow impaction zone (small, $<50 \%$ of the aneurysm area; large, $>50 \%$ of the aneurysm area), 3) the inflow jet concentration (concentrated or diffuse), 4) the flow pattern type (simple, single vortical structure; or complex, $>1$ vortical structure). 5) terminal flow pattern type (type A, flow splits from the parent vessel into the 2 daughter branches and the aneurysm; type B, flow splits into 1 daughter branch and the aneurysm; type C, flow enters the aneurysm before flowing to the daughter branches). It was verified that the main aneurysm hemodynamic characteristics did not change by varying the inflow conditions for different heart rates. In addition, the maximum WSS at the neck and the aneurysm sac was recorded, and the area of the aneurysm under low WSS was calculated. The maximum, minimum, and mean WSS of the aneurysm sac was recorded.

\section{Results}

\section{WSS and Pressure}

Visualizations of the distributions of hemodynamic forces (WSS and pressure) on the vascular wall at peak systole are presented in Fig 3. These visualizations show that the WSS (Fig 3 , left panel) reached a maximum of $393 \mathrm{dyne} / \mathrm{cm}^{2}$ at the neck of the aneurysm and the origin of the left posterior cerebral artery. In the aneurysm sac, the WSS reached a maximum of $160 \mathrm{dyne} / \mathrm{cm}^{2}$ on the left part of the aneurysm body at the zone of impaction of the inflow stream. The size of the flow impingement region (and elevated WSS) was small compared with the aneurysm sac (approximately 20\% of the area of the aneurysm sac). The rest of the aneurysm sac was subjected to WSS values lower than those in the parent basilar artery. The pressure was mostly uniform throughout the aneurysm sac, except for a small region around the point of flow impaction, where a small overpressure of approximately $1.5 \mathrm{~mm} \mathrm{Hg}$ could be observed (Fig 3, right panel). The minimum and mean WSS within the aneurysm sac was $0.13 \mathrm{dyne} / \mathrm{cm}^{2}$ and 14.7 dyne/ $\mathrm{cm}^{2}$, respectively.

\section{Intra-Aneurysmal Flow Pattern}

Visualizations of the intra-aneurysmal blood flow structure at peak systole are presented in Fig 4. This figure shows instantaneous streamlines colored with the velocity magnitude, from 2 viewpoints. The flow stream from the parent vessel splits at the left part of the aneurysm neck, creating 2 streams, 1 flowing to the left posterior cerebral artery and the other into the aneurysm. The stream flowing into the aneurysm impacts the aneurysm wall on the left side of the aneurysm body and creates a complex flow pattern with several regions of flow recirculation before flowing into the right posterior cerebral artery.

\section{Inflow Stream}

The inflow stream was visualized by using isovelocity surfaces and cutting planes. The velocity isosurface corresponding to $\mathrm{v}=40 \mathrm{~cm} / \mathrm{s}$ is presented in Fig 5 (left column) from 2 viewpoints. The velocity magnitude distributions on 2 selected cut- 


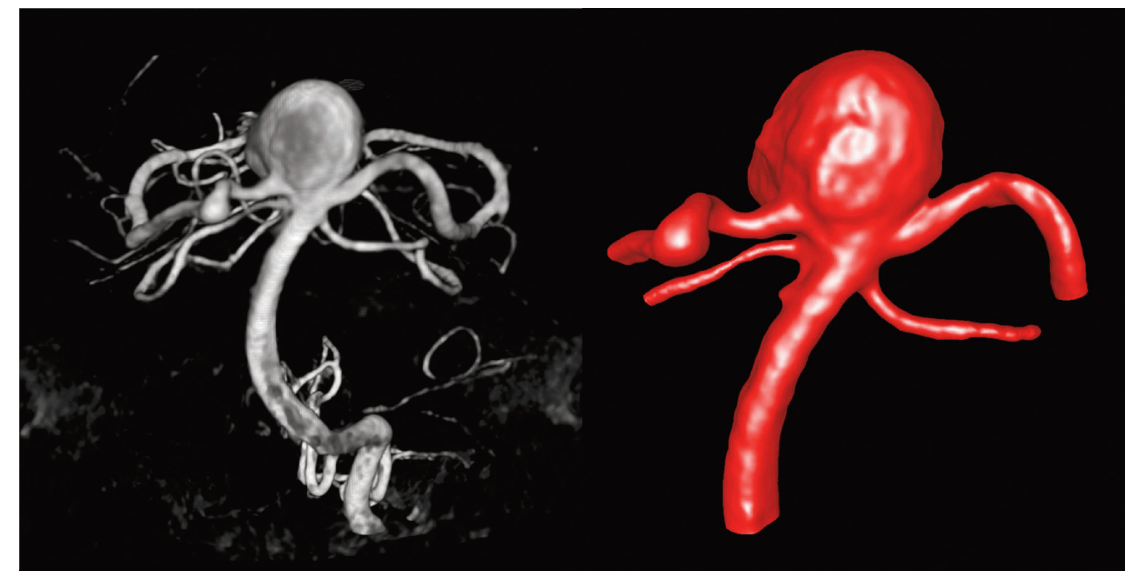

Fig 2. Anteroposterior view of the 3DRA of the basilar artery aneurysm before its rupture (left) and the corresponding geometric model (right).

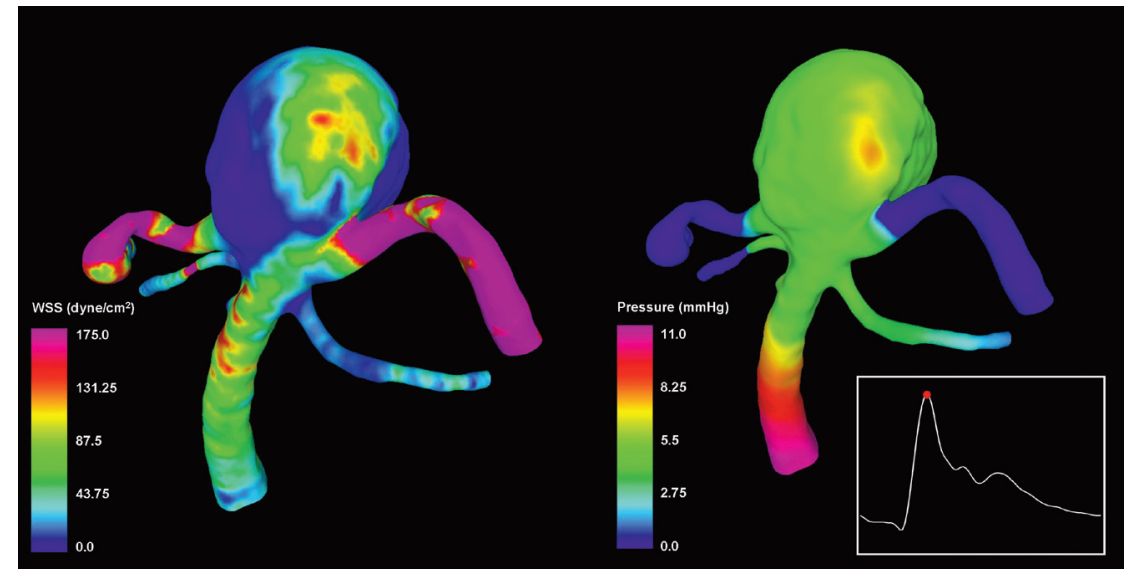

Fig 3. Visualizations of the distributions of WSS magnitude (left) and pressure (right) at peak systole.

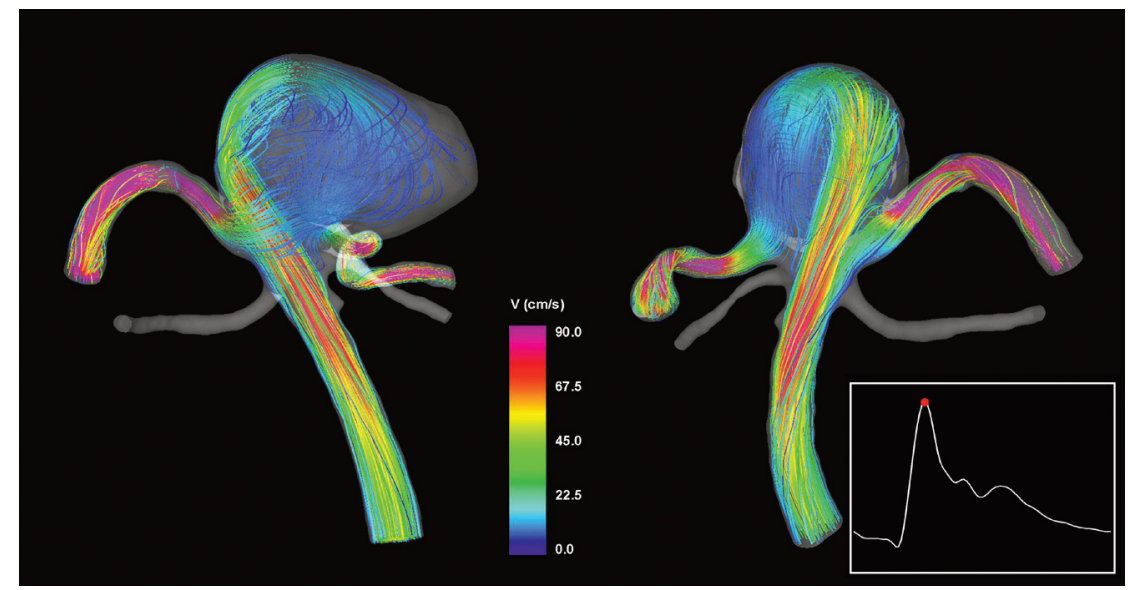

Fig 4. Visualizations of the aneurysm blood flow pattern at peak systole from 2 viewpoints. Streamlines are rendered with colors according to the velocity magnitude

ting planes are also shown in Fig 5 (right column). The locations of the cutting planes are shown together with the isovelocity surfaces. These visualizations reveal a fairly concentrated inflow jet that impacts a relatively small region of the aneurysm wall. After impacting the wall, the inflow jet disperses into a wide umbrella-shaped structure along the aneurysm wall, filled with slower vortical structures toward the center of the aneurysm volume.

\section{Discussion}

Previous studies relating hemodynamics and aneurysm rupture have identified some hemodynamic characteristics as potential rupture risk indicators. These studies, along with studies relating geometric properties of aneurysms and rupture, ${ }^{16-19}$ were based on the assumption that the rupture event did not substantially affect the aneurysm geometry and, therefore, the hemodynamic patterns obtained were represen- 


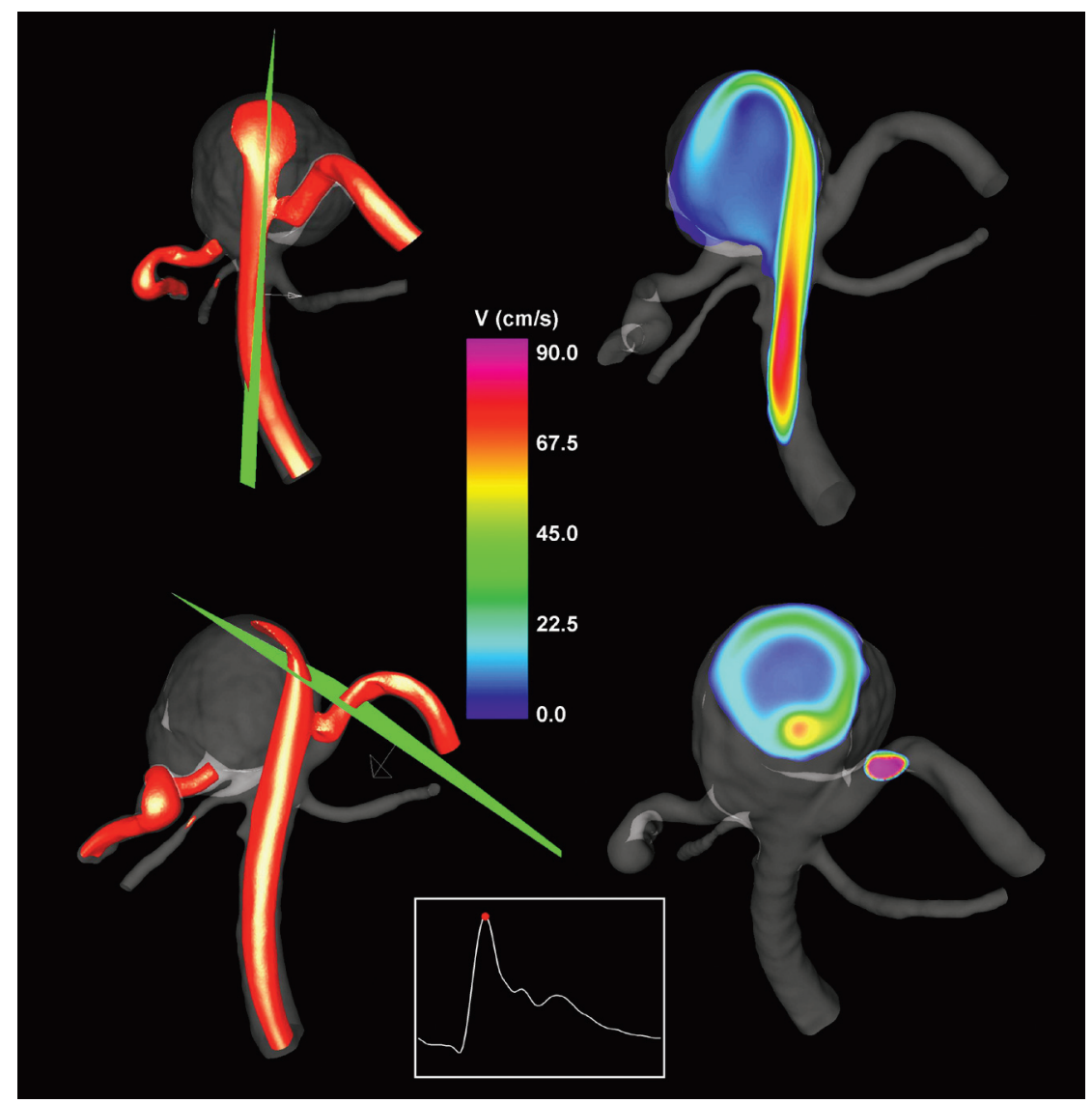

Fig 5. Visualizations of the inflow jet into the aneurysm by using isovelocity surfaces (left column) at peak systole and velocity magnitudes on plane cuts through the inflow jet (right). The locations of the cutting planes are displayed together with the velocity isosurfaces (left).

tative of the blood flow structures before the rupture. This assumption is common to most studies of aneurysm rupture because unruptured aneurysms are typically treated before they rupture. Thus, it is very difficult to connect the proposed risk indices to the rupture of an individual aneurysm. In the case presented here, we had the rare opportunity of analyzing the hemodynamics in an aneurysm just before its rupture and testing whether this analysis would place this particular aneurysm in the high-risk categories. Indeed it was observed that this aneurysm had a concentrated inflow jet impacting a small region of the aneurysm wall, creating a small region of locally elevated WSS near the impaction zone with most of the aneurysm sac under low WSS and a complex flow pattern inside the aneurysm. These characteristics were previously associated with aneurysm rupture. Additionally, the aneurysm morphology was of the terminal type; thus, it could also be classified according to how the flow stream from the parent vessel divides into the aneurysm and the daughter branches. It was found that the flow stream from the parent vessel split into 2 streams, 1 flowing to 1 of the daughter branches and the other into the aneurysm before flowing to the other daughter branch. This flow division structure has also been previously associated with aneurysm rupture. Finally, the maximum WSS observed in the aneurysm sac $\left(160 \mathrm{dyne} / \mathrm{cm}^{2}\right)$ was closer to the mean WSS in ruptured $\left(188 \mathrm{dyne} / \mathrm{cm}^{2}\right)$ than to unruptured (118 dyne/ $\mathrm{cm}^{2}$ ) aneurysms of the terminal morphologic type previously studied. All these observations would then designate this aneurysm as at high risk of rupture, and indeed, it did go on to bleed. These results are, therefore, consistent with previous studies relating hemodynamics and aneurysm rupture and support the notion that hemodynamic characteristics may be used to help stratify the rupture risk of cerebral aneurysms.

Recently, Cebral et al reported a similar study. ${ }^{20}$ In that case, the hemodynamic characteristics in a basilar artery aneurysm just before its rupture were described. These characteristics also included a concentrated inflow jet impacting a small region of the aneurysm wall with an associated complex flow pattern and a region of elevated WSS around the impaction point. However, that aneurysm was fusiform and had a mild stenosis just upstream of the aneurysm, which concentrated the flow stream at the aneurysm inlet. The previous studies relating hemodynamics and aneurysm rupture considered mainly saccular aneurysms ${ }^{4}$ and focused on the terminal morphologic subtype ${ }^{6}$ and on a subsample of aneurysms located at the anterior communicating artery. ${ }^{5}$ In contrast to the study of Cebral et $\mathrm{al}^{20}{ }^{20}$ the current study analyzes the hemodynamic environment just before the rupture of a saccular aneurysm with a terminal morphology. Thus, this study adds confirmation that the hemodynamic patterns described are consistent with those most commonly found in aneurysms with a previous history of bleeding.

The current study, however, does not provide information about the actual rupture site or its underlying mechanism. Unfortunately, no pathologic information was available from this case to identify the rupture site and help correlate the 
hemodynamic condition mode specifically to it. This study only provides information about the hemodynamic environment of the aneurysm just before its rupture. Although the characteristics of this hemodynamic environment have been observed to occur more often in aneurysms with a previous history of bleeding, this does not demonstrate that these characteristics are the cause of the rupture. In particular, the local pressure increase at the impingement zone is small compared with the normal intra-arterial pressure variation during the cardiac cycle and may not explain the rupture. Other factors may be involved in the process of rupture such as systemic hypertension or connective tissue deficiencies. Contacts from the perianeurysmal environment may cause abnormal damaging stresses and strains related to the vascular pulsatility.

Finally, the current study has several limitations common to most CFD analyses, which should be considered when evaluating the results. There are several sources of error that can affect the accuracy of the numeric simulations. The vascular geometry was approximated from 3D angiography image data and may have distortions from the process of construction and image optimization. The flow conditions were not patientspecific but were derived from measurements on healthy subjects. The inflow profile was assumed fully developed from representative measurements on healthy patients. Several assumptions were made for the CFD calculations, including outflow boundary conditions, rigid walls, and Newtonian properties. The wall biomechanics and perianeurysmal environment were not considered. However, CFD results have been shown to be robust to small variations of many of these assumptions and approximations ${ }^{7}$ and to be able to realistically represent the in vivo hemodynamics. ${ }^{21}$ The model construction and meshing took only a few minutes, and the pulsatile simulation ran in approximately 12 hours on a single core of a silicon graphics ICE cluster (http://www.sgi.com/ products/servers/altix/ice) with 2 quad Xeon $5440 @ 2.83 \mathrm{GHz}$ per node and $16 \mathrm{~GB}$ of RAM per node. It was also verified that a steady-flow calculation, which was completed in 2 minutes, provided similar qualitative information about the flow characteristics. This suggests that these techniques can potentially be used routinely for patient evaluation during angiography examinations.

\section{Conclusions}

This hemodynamic analysis of a saccular intracranial aneurysm of the terminal morphologic subtype that ruptured a few hours after being imaged is consistent with previous studies correlating aneurysm rupture and hemodynamic patterns in saccular and terminal aneurysms. This study adds further support to the notion that hemodynamic information may be used to help stratify the rupture risk of cerebral aneurysms.

\section{References}

1. Kassell NF, Torner JC, Haley EC, et al. The International Cooperative Study on the Timing of Aneurysm Surgery. Part 1. Overall management results. J Neurosurg 1990;73:18-36

2. Nishioka H, Torner JC, Graf CJ, et al. Cooperative study of intracranial aneurysms and subarachnoid hemorrhage: a long-term prognostic study. II. Ruptured intracranial aneurysms managed conservatively. Arch Neurol 1984;41:1142-46

3. White PM, Wardlaw JM. Unruptured intracranial aneurysms. J Neuroradiol 2003;30:336-50

4. Cebral JR, Castro MA, Burgess JE, et al. Characterization of cerebral aneurysms for assessing risk of rupture by using patient-specific computational hemodynamics models. AJNR Am J Neuroradiol 2005;26:2550-59

5. Castro MA, Putman CM, Sheridan MJ, et al. Hemodynamic patterns of anterior communicating artery aneurysms: a possible association with rupture. AJNR Am J Neuroradiol 2009;30:297-302

6. Castro M, Putman C, Radaelli A, et al. Hemodynamics and rupture of terminal cerebral aneurysms. Acad Radiol 2009;16:1201-7. Epub 2009 Jun 23

7. Cebral JR, Castro MA, Appanaboyina S, et al. Efficient pipeline for imagebased patient-specific analysis of cerebral aneurysm hemodynamics: technique and sensitivity. IEEE Trans Med Imaging 2005;24:457-67

8. Yim PJ, Vasbinder GB, Ho VB, et al. Isosurfaces as deformable models for magnetic resonance angiography. IEEE Trans Med Imaging 2003;22:875-81

9. Taubin G. A signal processing approach to fair surface design. In: Proceedings of the 22nd Annual Conference on Computer Graphics and Interactive Techniques, Los Angeles, California, January 18-25, 1995:351-58

10. Kundu PK, Cohen IM. Fluid Mechanics. New York: Academic Press (Elsevier); 2004

11. Cebral JR, Yim PJ, Löhner R, et al. Blood flow modeling in carotid arteries using computational fluid dynamics and magnetic resonance imaging. Acad Radiol 2002;9:1286-99

12. Löhner R, Yang C, Cebral JR, et al. Fluid-structure-thermal interaction using adaptive unstructured grids. In: Kvamsdal, ed. Computational Methods for Fluid-Structure Interaction. Toronto, Ontario, Canada: Tapir Press; 1999:109-20

13. Womersley JR. Method for the calculation of velocity, rate of flow and viscous drag in arteries when the pressure gradient is known. J Physiol 1955;127:553-63

14. Cebral JR, Castro MA, Putman CM, et al. Flow-area relationship in internal carotid and vertebral arteries. Physiol Meas 2008;29:585-94

15. Valencia A, Guzman AM, Finol EA, et al. Blood flow dynamics in saccular aneurysm models of the basilar artery. J Biomech Eng 2006;128:516-26

16. Ujiie $H$, Tachibana $H$, Hiramtsu O. Effects of size and shape (aspect ratio) on the hemodynamics of saccular aneurysms: a possible index for the surgical treatment of intracranial aneurysms. Neurosurgery 1999;45:119-30

17. Ma B, Harbaugh RE, Raghavan ML. Three-dimensional geometrical characterization of cerebral aneurysms. Ann Biomed Eng 2004;32:264-73

18. Raghavan ML, Ma B, Harabaugh RE. Quantified aneurysm shape and rupture risk. J Neurosurg 2005;102:355-62

19. Millan D, Dempere-Marco L, Pozo JM, et al. Morphological characterization of intracranial aneurysms using 3-D moment invariants. IEEE Trans Med Imaging 2007;26:1270-82

20. Cebral JR, Hendrickson S, Putman CM. Hemodynamics in a lethal basilar artery aneurysm just before its rupture. AJNR Am J Neuroradiol 2009;30:95-98

21. Cebral JR, Pergolizzi R, Putman CM. Computational fluid dynamics modeling of intracranial aneurysms: qualitative comparison with cerebral angiography. Acad Radiol 2007;14:804-13 